\title{
PÓS-COLHEITA DE MANGAS 'TOMMY ATKINS' RECOBERTAS COM QUITOSANA ${ }^{1}$
}

\author{
MARIA LUZENIRA DE SOUZA², CRISTIANE MARIA ASCARI MORGADO ${ }^{3}$, \\ KELLY MAGALHÃES MARQUES ${ }^{3}$, CLAUDIA FABRINO MACHADO MATTIUZ ${ }^{4}$, \\ BEN-HUR MATTIUZ ${ }^{5}$
}

RESUMO - O objetivo deste trabalho foi avaliar a influência de cobertura de quitosana na pós-colheita de mangas 'Tommy Atkins', colhidas "de vez" e armazenadas a $23{ }^{\circ} \mathrm{C}(65 \% \mathrm{UR})$. As mangas foram adquiridas na CEAGESP de Ribeirão Preto-SP, e transportadas ao Laboratório de Tecnologia de Produtos Agrícolas da UNESP de Jaboticabal. Após a seleção, os frutos foram higienizados em solução de dicloro s. triazina-

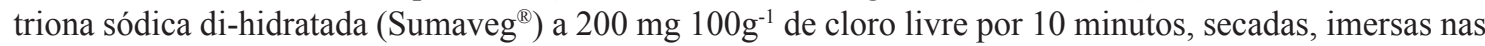
soluções a $0 \% ; 1,0 \% ; 1,5 \%$ e $2,0 \%$ de quitosana por 1 minuto, secadas sob ventilação, acondicionadas em bandejas e armazenadas a $23 \pm 2{ }^{\circ} \mathrm{C}$ e $65 \pm 5 \%$ UR, por 9 dias. Foram utilizados três repetições com dois frutos cada. Avaliaram-se, a cada três dias, a perda de massa fresca, a cor, a firmeza, os teores de ácido ascórbico, de sólidos solúveis, de acidez titulável e sólidos solúveis / acidez titulável. O recobrimento com quitosana retarda o amadurecimento de mangas 'Tommy Atkins' "de vez", durante nove dias de armazenamento a 23 ${ }^{\circ} \mathrm{C}$, sendo que a concentração de $1,5 \%$ propicia melhor manutenção da cor da polpa, dos teores de sólidos solúveis, de acidez titulável, de ácido ascórbico, dos valores de SS/AT e de firmeza.

Termos para indexação: Mangifera indica, conservação, qualidade.

\section{POST-HARVEST OF 'TOMMY ATKINS' MANGOES COVERED WITH CHITOSAN}

\begin{abstract}
The objective of this work was to evaluate the influence of chitosan in the post-harvest of 'Tommy Atkins' mangoes, harvested in the semi-ripe stage and stored at $23^{\circ} \mathrm{C}(65 \% \mathrm{RH})$. Mangoes from CEAGESP, Ribeirão Preto, SP, Brazil were transported to the Laboratory of Technology of Agricultural Products of UNESP Jaboticabal. After selection, the fruits were washed and the surface sanitized with a

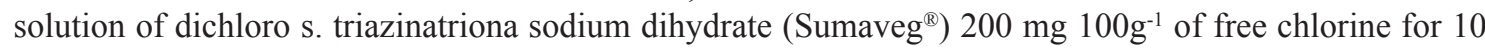
minutes, dried, dipped in solution $0 \% ; 1.0 \% ; 1.5 \% ; 2.0 \%$ oh chitosan for 1 minute, dried under ventilation, packed in trays and stored at $23 \pm 2{ }^{\circ} \mathrm{C}$ and $65 \pm 5 \%$ UR for 9 days. Three replicates of two fruits each were used. The fruits every three days had the weight loss, the color, the firmness, the ascorbic acid content, the soluble solids, the titratable acidity and the soluble solids / titratable acidity evaluated. The coating with chitosan delayed the ripening of 'Tommy Atkins' semi-ripe mangoes stored at $23^{\circ} \mathrm{C}$. The concentration of $1.5 \%$ provided better maintenance of color, firmness, ascorbic acid content, soluble solids, titratable acidity and soluble solids / titratable acidity
\end{abstract}

Index terms: Mangifera indica, conservation, quality.

\footnotetext{
${ }^{1}$ Trabalho Sinfruit 134 - Simpósio Internacional de Fruticultura - Avanços na Fruticultura (17 a 21 Outubro)

${ }^{2}$ Profa. Associada da Universidade Federal do Acre/UFAC, BR 364, km 4. CEP: 69910-630. Rio Branco-AC. E-mail:mluzen@hotmail.com ${ }^{3}$ Alunas do Programa de Produção Vegetal da FCAV-UNESP, Câmpus de Jaboticabal. Via de Acesso Prof. Paulo Donato Castellane, s/n. CEP: 14.884-900. Jaboticabal-SP. E-mail: cristianemorgado4@yahoo.com.br; kelly_mgmq@hotmail.com

${ }^{4}$ Profa. Dra. do Centro Universitário Moura Lacerda. Av. Dr. Oscar de Moura Lacerda, 1520. CEP: 14076-510. Ribeirão Preto-SP. E-mail: cmattiuz@gmail.com

${ }^{5}$ Prof. Dr. da FCAV-UNESP, Câmpus de Jaboticabal, Departamento de Tecnologia. Via de Acesso Prof. Paulo Donato Castellane, s/n. CEP: 14.884-900. Jaboticabal-SP. E-mail: benhur@fcav.unesp.br
} 


\section{INTRODUÇ̃̃O}

A manga (Mangifera indica, L.) é um fruto tipicamente tropical, com sabor e aroma muito atrativos, que conquistou a preferência de consumidores e apresenta, hoje, expressividade positiva no agronegócio internacional. Em 2010, no período de janeiro até abril, o volume exportado foi em torno de 45.200 toneladas (AGRIANUAL, 2011), demonstrando um grande potencial do País como exportador de manga in natura.

Para garantir maior competitividade no mercado de exportação de frutas, é necessário produzir em épocas adequadas à comercialização, assim como garantir a qualidade de frutos que atendam aos padrões internacionais de segurança alimentar (HOJO et al., 2009). Desse modo, o emprego de técnicas de conservação, utilizando produtos naturais que prolonguem a vida útil pós-colheita, é importante, visto que a manga tem vida útil limitada.

Pesquisas vêm sendo realizadas utilizando filmes e revestimentos que apresentam o potencial para retardar as reações de degradação e/ou síntese de substâncias em frutas, proporcionado maior vida útil, como, por exemplo, em mangas (AMARIZ et al., 2010; PLOTTO et al., 2010) e goiabas (CERQUEIRA et al., 2011).

A quitosana, uma forma deacetilada da quitina, é um polissacarídeo de alta massa molecular, solúvel em ácidos orgânicos, é comestível e segura para os seres humanos. Apresenta efeito fungicida, além de ser um material natural e não tóxico (BAUTISTA-BANÕS et al., 2006). Oferece um promissor e versátil polímero biodegradável para embalagens de alimentos, podendo ser usado como biocatalisadores em embalagem de alimentos (DUTTA et al., 2007).

$\mathrm{O}$ tratamento de tomates com quitosana em cobertura, armazenados a $25^{\circ} \mathrm{C}$ e $2{ }^{\circ} \mathrm{C}$, induziu aumento significativo na atividade das enzimas polifenoloxidase e peroxidase, e no conteúdo de compostos fenólicos, além de promover ação fungistática e elicitora contra patógenos (LIU et al., 2007).

Como efeitos positivos da quitosana, além da atividade antimicrobiana direta, estudos sugerem que ela induz fortemente uma série de reações de defesa correlacionada com atividades enzimáticas, sendo indicada para aumentar a produção de glucano-hidrolases, compostos fenólicos e síntese de fitoalexinas específicas, além de reduzir a atividade das enzimas poligalacturonases e pectinametilesterases, responsáveis pelo amaciamento (BAUTISTA-BAÑOS et al., 2006).

Atualmente, tem sido dada mais atenção ao uso de materiais contendo biomoléculas ativas com propriedades antimicrobianas que possam melhorar a segurança alimentar, elevar a vida útil dos produtos e não provocar impacto negativo ao meio ambiente (DUTTA et al., 2007). Desse modo, o uso de substâncias naturais, a exemplo da quitosana, aplicada como cobertura na pós-colheita de frutas, pode ser uma alternativa viável para elevar a vida pós-colheita de manga 'Tommy Atkins' sem o uso da refrigeração, processo oneroso, contribuindo para a redução dos custos ao longo da cadeia produtiva.

Neste contexto, o objetivo deste trabalho foi avaliar a influência de cobertura à base de quitosana na vida pós-colheita de mangas 'Tommy Atkins', colhidas "de vez".

\section{MATERIAL E MÉTODOS}

Mangas 'Tommy Atkins', foram adquiridas na CEAGESP do município de Ribeirão Preto-SP, no estádio de maturação fisiológico (“de vez"), correspondentes à cor da casca no grau 3 da escala de coloração da GTZ (1992).

As mangas foram transportadas ao Laboratório de Tecnologia de Produtos Agrícolas da FCAV-UNESP, Câmpus de Jaboticabal, onde foram novamente selecionadas, reduzindo-se os pedúnculos para $20 \mathrm{~mm}$. Na sequência, foram lavadas com detergente, enxaguadas com água potável, higienizadas em solução de dicloro $\mathrm{s}$. triazinatriona sódica di-hidratada (Sumaveg ${ }^{\circledR}$ ) a $200 \mathrm{mg} 100 \mathrm{~g}^{-1}$ de cloro livre, por 10 minutos, secas ao ambiente e separadas em lotes, correspondentes aos tratamentos.

A quitosana comercial utilizada (densidade de $0,6 \mathrm{~g} \mathrm{~mL}^{-1}$, viscosidade de 20 a $50 \mathrm{cps}$ e grau de desacetilização de $90 \%$ ) foi dissolvida em água, com auxílio de $1 \%$ de ácido láctico, para obter as concentrações de $1,0 \%, 1,5 \%$ e $2,0 \%$. Em cada solução, foram adicionados $3 \%$ de glicerol, como plastificante, e ajustou-se o $\mathrm{pH}$ para 5,6 com $\mathrm{NaOH}$ $0,1 \mathrm{M}$. Os tratamentos consistiram em imergir, por um minuto, as mangas nas soluções de quitosana: [1] Controle - sem imersão em solução de quitosana; [2] Quitosana a 1,0\%; [3] Quitosana a 1,5\%; [4] Quitosana a 2,0\%. Após a aplicação da quitosana, aguardou-se a cobertura secar e, em seguida, as frutas foram armazenadas a $23 \pm 2{ }^{\circ} \mathrm{C}$ e $65 \pm 5 \% \mathrm{UR}$, por nove dias.

A massa fresca foi quantificada, utilizando-se de balança digital com capacidade para $2.000 \mathrm{~g}$ e precisão de $0,1 \mathrm{~g}$. A coloração da polpa foi obtida, realizando-se o corte das frutas no momento da análise e medindo-se a coloração da polpa com o uso do colorímetro Minolta Corp CR-400, sendo expressa pelo ângulo de cor (Hue). A firmeza da polpa foi ava- 
liada através do penetrômetro FT 327, com ponteira de $8 \mathrm{~mm}$, sendo os resultados expressos em Newton. O teor de sólidos solúveis (SS), expresso em ${ }^{\circ}$ Brix; o de acidez titulável (AT), expresso em \% de ácido

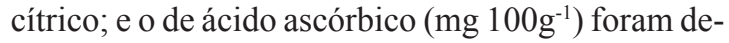
terminados conforme metodologia da AOAC (1997).

$\mathrm{O}$ delineamento experimental foi o inteiramente casualizado, em esquema fatorial composto por dois fatores: quitosana $(0 ; 1,0 \%, 1,5 \%$ e $2,0 \%)$ e dias de armazenamento $(0 ; 3 ; 6$ e 9 dias). Foram utilizadas três repetições, com dois frutos cada. Os dados obtidos foram submetidos à análise de variância, pelo teste $\mathrm{F}$, e as médias foram comparadas pelo teste de Tukey $(P<0,05)$, em que as diferenças entre dois tratamentos maiores que a soma de dois desvios-padrão foram consideradas significativas.

\section{RESULTADOS E DISCUSSÃO}

As mangas apresentaram perda acumulada de massa fresca de 3,5\% ao longo do armazenamento (Figura 1), sendo que o tratamento de quitosana a $1 \%$ proporcionou melhor manutenção da massa fresca $(3,28 \%)$, e os frutos não tratados foram os que perderam mais massa $(3,8 \%)$. Cabe salientar que a diferença média entre os tratamentos foi muito pequena $(0,51 \%)$. O uso de quitosana na conservação pós-colheita de pêssegos 'Douradão' não foi eficiente em reduzir a perda de massa dos frutos (SANTOS et al., 2008), enquanto Jiang e Li (2001) relataram que frutos de longan (Dimocarpus longan Lour), também conhecido como "olho-do-dragão", tratados com cobertura de quitosana a 0,$5 ; 1,0$ e $2,0 \%$, armazenados a $2{ }^{\circ} \mathrm{C}(90 \%$ UR) durante 30 dias, apresentaram reduções na taxa respiratória, perda de massa, atividade da enzima polifenoloxidase, mudança de cor, manteve a qualidade comestível e inibiu parcialmente a deterioração durante a estocagem. Segundo Chitarra e Chitarra (2005), perdas de massa da ordem de 3 a $6 \%$ são suficientes para causar um marcante declínio na qualidade, mas alguns produtos são ainda comercializáveis com $10 \%$ de perda de umidade.

Estes dados corroboram os de Sun-Hua et al. (2007), que demonstraram que o uso de cobertura com $1,5 \%$ de quitosana, $0,10 \%$ de preservativo e pH 5,45 foi a combinação ideal para a conservação de mangas, na temperatura de $26-30{ }^{\circ} \mathrm{C}$, mantendo a firmeza por mais de 18 dias, inibindo a microbiota e proporcionando menor perda de massa fresca.

$\mathrm{O}$ ângulo de cor (Hue) manteve-se nas mangas recobertas com quitosana, e apesar de não apresentarem diferenças significativas, a polpa dos frutos-controle apresentou redução dos valores ao longo do armazenamento (Figura 2). A redução do ângulo de cor no primeiro quadrante $\left(0^{\circ}\right.$ a $\left.90^{\circ}\right)$ indica que a cor de um produto migra do amarelo para o vermelho. Neste caso, a redução de $89^{\circ}$ para $83^{\circ}$, nos frutos-controle, indica que a polpa se tornou progressivamente mais amarelada ao longo do armazenamento, sinalizando um amadurecimento mais rápido desses frutos, quando comparados aos demais tratamentos. A quitosana forma um filme semipermeável ao redor dos frutos que reduz as trocas gasosas com o ambiente, levando à modificação da atmosfera no interior do fruto (BAUTISTA-BANÕS et al., 2006), promovendo bloqueio do processo de síntese de carotenoides ou de degradação da clorofila. Tal efeito, provavelmente, confirma a inibição dos eventos metabólicos que viabilizam a mudança da cor da polpa nas mangas, reduzindo, assim, o amadurecimento. Djioua et al. (2010) também observaram em mangas 'Tommy Atkins' minimamente processadas, tratadas com cobertura de quitosana a $0,25 \%$ e armazenadas a $6^{\circ} \mathrm{C}$, por nove dias, que a quitosana impediu a alteração da cor durante o armazenamento. Em relação à firmeza, observou-se que houve redução desta ao longo do armazenamento, com valores médios iniciais de $127,12 \mathrm{~N}$ e $10,87 \mathrm{~N}$ após nove dias a $23{ }^{\circ} \mathrm{C}$ (Figura 3). O melhor resultado foi verificado no tratamento de quitosana a $1,5 \%$, com valor médio de $53,43 \mathrm{~N}$, e o pior resultado foi atribuído às mangas não tratadas (média de 44,24 N). Estes resultados corroboram os encontrados por Zhu et al. (2008), que relataram a eficácia do revestimento de quitosana no retardamento do amadurecimento de manga 'Tainong,' com retenção da firmeza, atraso na mudança de cor e inibição da taxa respiratória.

Em mamão minimamente processado, o revestimento com quitosana manteve a cor e a firmeza atrativas, juntamente com baixa carga microbiana, mostrando-se boa alternativa para a preservação da qualidade e aumento da vida útil (GONZÁLEZ-AGUILAR et al., 2009).

Os teores de ácido ascórbico foram influenciados significativamente pelos tratamentos (Figura 4). Notou-se que o conteúdo desse ácido nos frutos-controle sofreu grande redução, diferindo significativamente dos demais. Dentre os tratados com quitosana, a concentração de $1,5 \%$ foi a que propiciou melhor manutenção do conteúdo desse ácido, indicando retardo na senescência dos frutos. Segundo Chitarra e Chitarra (2005), o ácido ascórbico é um composto antioxidante sintetizado pelas frutas e hortaliças em quantidades variáveis, de acordo com a espécie, cultivar, fatores ambientais, nutricionais e grau de maturação. Sendo assim, os valores de vitamina $\mathrm{C}$ tendem a diminuir com o amadurecimento devido à atuação direta da enzima ácido 
ascórbico oxidase (ascorbinase), além da oxidação e consequente transformação do ácido ascórbico em ácido 2,3-dicetogulônico.

Houve incremento do teor de sólidos solúveis (SS) ao longo do armazenamento, independentemente do tratamento (Figura 5A). Apesar de não ocorrerem diferenças significativas, verificou-se que, os frutos do tratamento-controle se destacaram com os maiores valores e que, no tratamento de quitosana a $1,5 \%$, apresentou os menores teores de sólidos solúveis. Percebeu-se que a quitosana interferiu no metabolismo das mangas, mantendo os teores de sólidos solúveis (açúcares, ácidos, outros) já sintetizados por ação do biopolímero, ao longo do tempo de estocagem. Zhu et al. (2008) enfatizaram a eficácia do revestimento de quitosana em manga 'Tainong', no retardamento do aumento de sólidos solúveis, corroborando os resultados deste trabalho.

Em relação à acidez titulável (AT), houve redução progressiva nos teores ao longo do período de armazenamento, em todos os tratamentos (Figura 5B). Observou-se que os menores valores ocorreram nas mangas não recobertas (Controle), diferindo significativamente dos tratamentos com quitosana. Essa é uma indicação de que os tratamentos com quitosana desaceleraram o processo normal de amadurecimento das mangas, visto que a diminuição da acidez em mangas está associada ao consumo de ácidos no processo respiratório, em decorrência da maturação (CHITARRA; CHITARRA, 2005). Morais et al. (2002) relataram que, durante o amadurecimento, as mangas tendem a aumentar o $\mathrm{pH}$ e diminuir a acidez. Estudos realizados em manga 'Tainong', por Zhu et al. (2008), confirmaram a eficácia do revestimento de quitosana na redução da acidez titulável.

No entanto, em estudos realizados por Chien et al. (2007), a quitosana como cobertura em manga minimamente processada retardou a perda de água, reduziu a qualidade sensorial, inibiu o crescimento de microrganismos e aumentou o teor de sólidos solúveis, da acidez titulável e o de ácido ascórbico. O efeito benéfico da aplicação da quitosana na redução da velocidade de amadurecimento das mangas pode ser verificado quando se analisam os valores da relação SS/AT, conhecida como ratio (Figura 5C). Observou-se que os valores para as mangas não tratadas com quitosana foram muito superiores às mangas tratadas, indicando amadurecimento acelerado destes frutos pelo aumento nos valores de sólidos solúveis (SS) e diminuição nos valores de acidez titulável (AT). A relação SS/AT é um dos índices mais utilizados para determinar a maturação e a palatabilidade dos frutos. Chitarra e Chitarra (2005) estabelecem que essa relação seja indicativa do sabor. Devido à característica biodegradável, não tóxica, de formar um revestimento semipermeável, prolongando a vida útil de frutas e vegetais após a colheita, a quitosana apresenta potencial para se tornar nova classe de protetor de vegetal, com o objetivo de auxiliar da agricultura sustentável (BAUTISTA-BAÑOS et al., 2006).

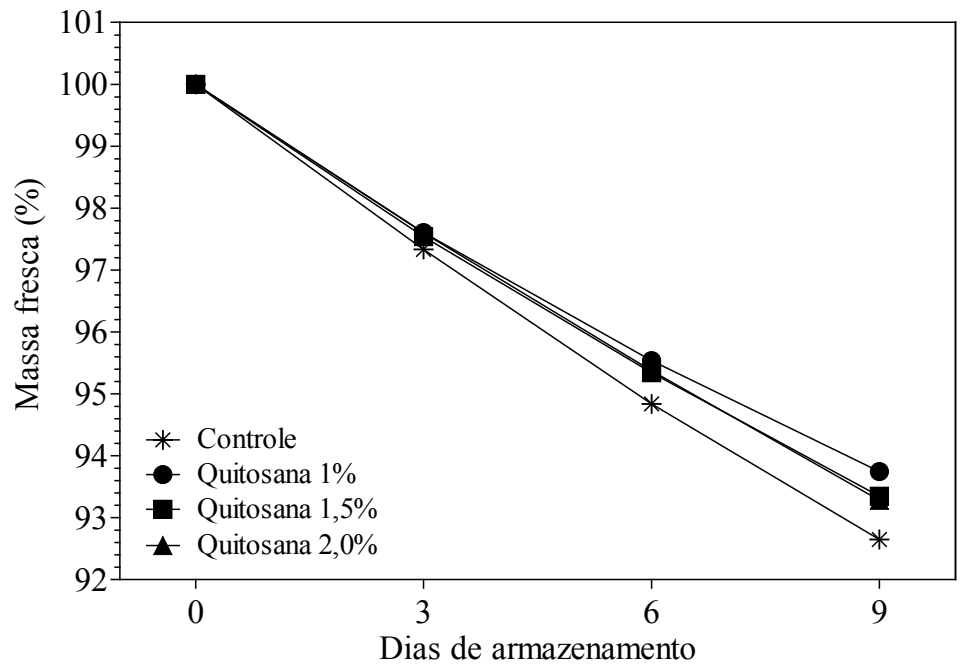

FIGURA 1 - Perda de massa fresca, em porcentagem, de mangas 'Tommy Atkins' “de vez”, tratadas com quitosana e armazenadas a $23{ }^{\circ} \mathrm{C}$. 


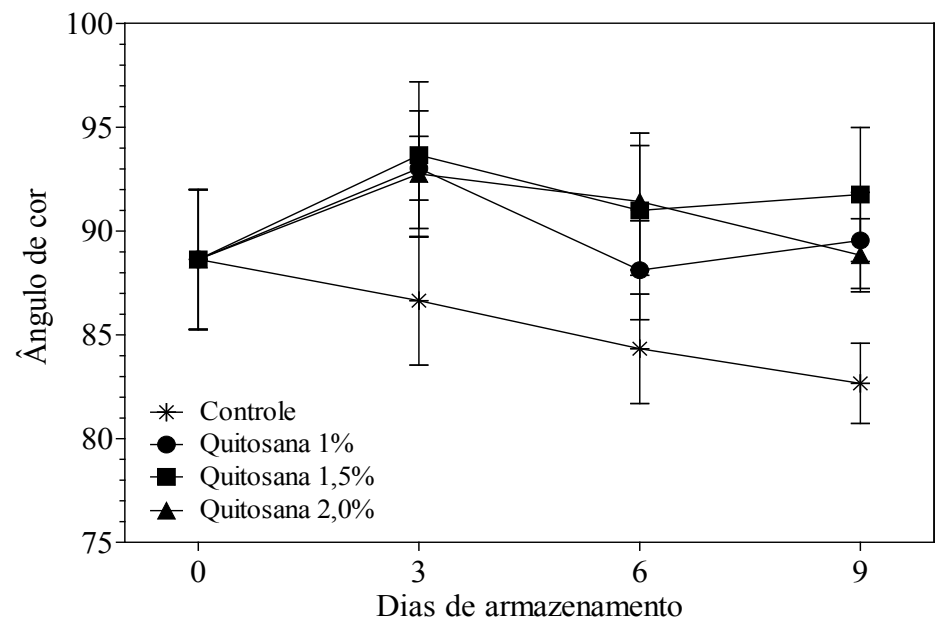

FIGURA 2 -Valores médios para ângulo de cor (Hue) de polpa de mangas “Tommy Atkins' "de vez”, tratadas com quitosana e armazenadas a $23^{\circ} \mathrm{C}$. Linhas verticais representam o desvio-padrão da média.

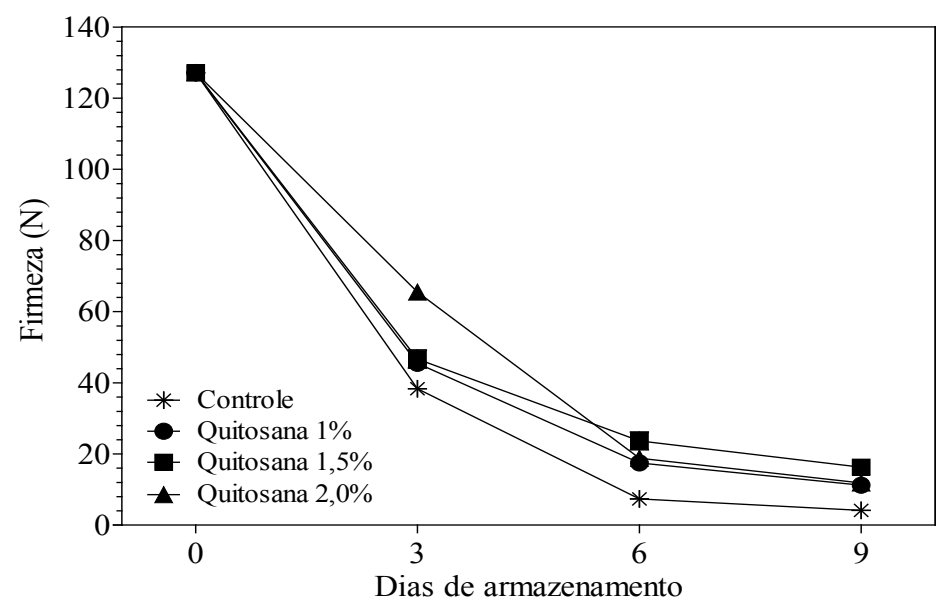

FIGURA 3 - Valores médios de firmeza (N), de mangas “Tommy Atkins' "de vez”, tratadas com quitosana e armazenadas a $23^{\circ} \mathrm{C}$.

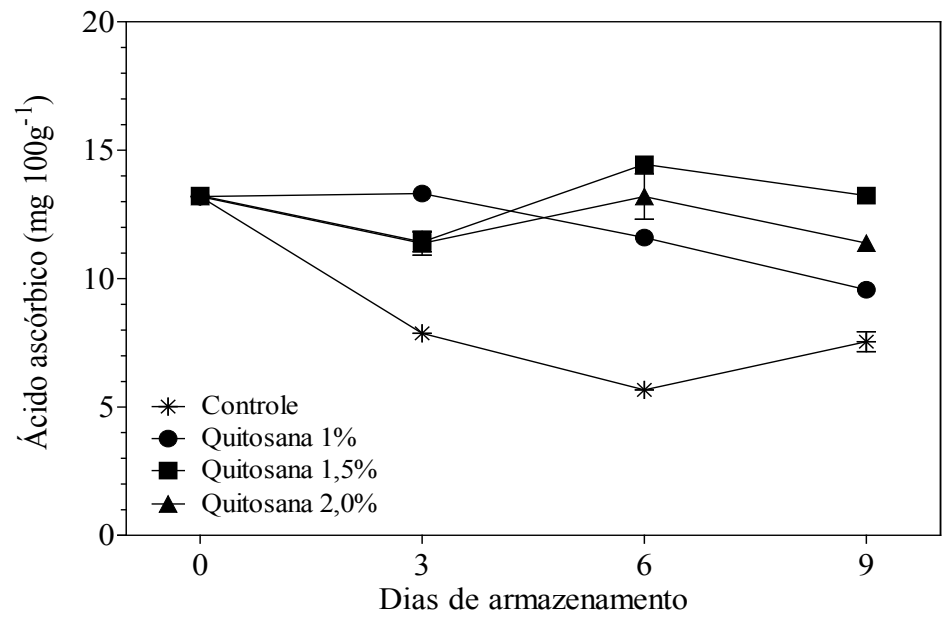

FIGURA 4 - Valores médios de ácido ascórbico, expressos em mg 100g-1, de mangas 'Tommy Atkins' “de vez", tratadas com quitosana e armazenadas a $23^{\circ} \mathrm{C}$. Linhas verticais representam o desvio-padrão da média. 


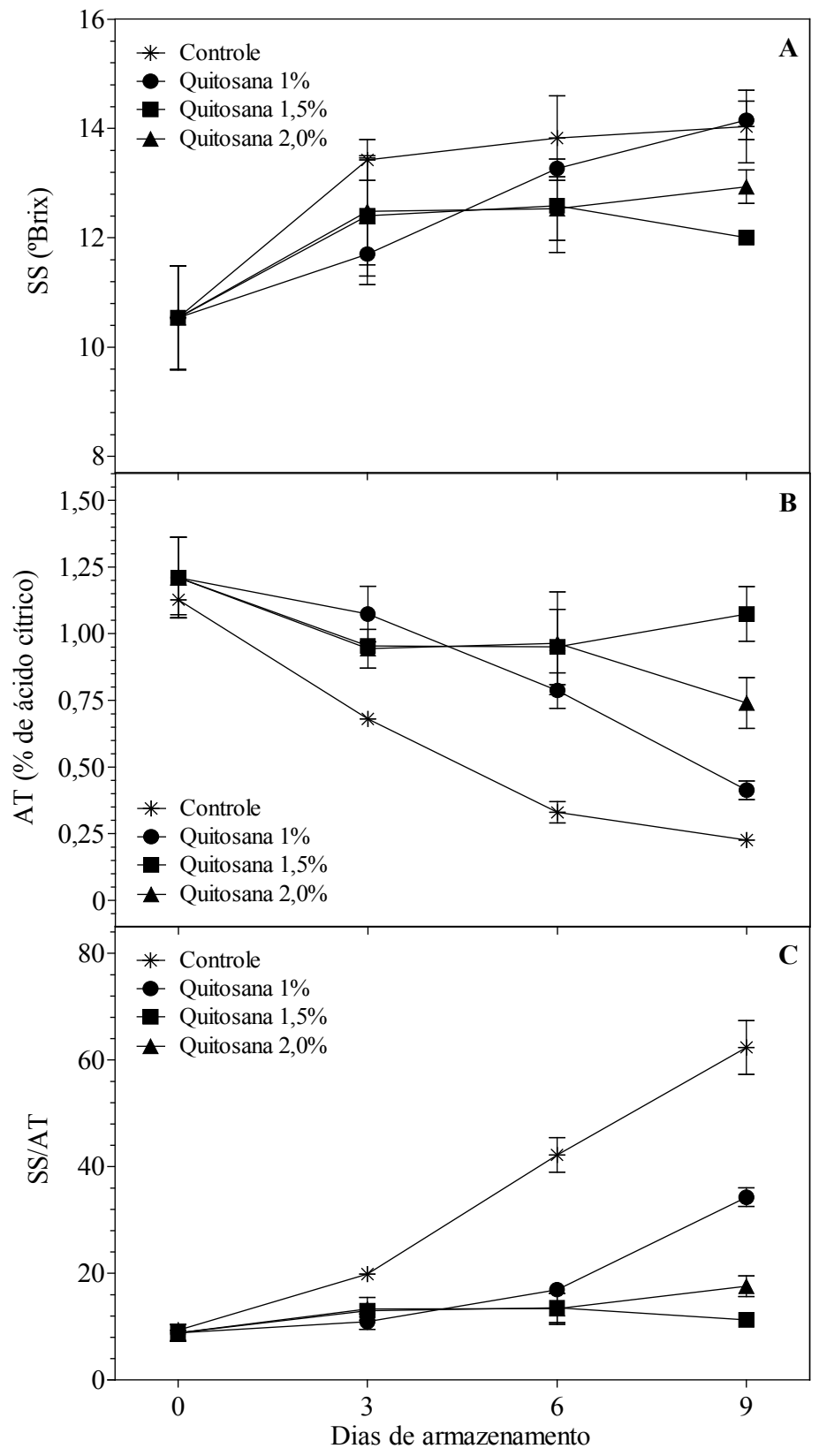

FIGURA 5 - Valores médios de sólidos solúveis (SS) (A), acidez titulável (AT) (B) e da relação SS/AT (C), de mangas 'Tommy Atkins' "de vez", tratadas com quitosana e armazenadas a $23{ }^{\circ} \mathrm{C}$. Linhas verticais representam o desvio-padrão da média.

\section{CONCLUSÕES}

O recobrimento com quitosana retarda o amadurecimento de mangas 'Tommy Atkins' "de vez", durante nove dias de armazenamento a $23{ }^{\circ} \mathrm{C}$, sendo que a concentração a $1,5 \%$ propicia melhor manutenção da cor da polpa, dos teores de sólidos solúveis, de acidez titulável, de ácido ascórbico, dos valores de SS/AT e de firmeza.

\section{REFERÊNCIAS}

AGRIANUAL: anuário da agricultura brasileira. São Paulo: FNP Consultoria \& Comercio, 2011. p. 338-344. 
AMARIZ, A.; LIMA, M. A. C de;TRINDADE, D. C. G da; SANTOS, A. C. N dos; RIBEIRO, T. P. Recobrimentos à base de carboximetilcelulose e dextrina em mangas 'Tommy Atkins' armzenadas sob refrigeração. Ciência Rural, Santa Maria, v.40, n.10, p.2199-2205, 2010.

A.O.A.C. Official methods of analysis of the Association of Official Analytical Chemists International. $16^{\text {th }}$ ed. Washington, 1997, v. 2. p. 37-45.

BAUTISTA-BANÕS, S.; HERNANDEZ-LAUZARDO, A. N.; VELAZQUEZ-DEL VALLE, M. G.; HERNÁNDEZ-LOPEZ, M.; BARKA, E. A.; BOSQUEZ-MOLINA, E.; WILSON, C. L. Chitosan as a potencial natural compound to control pre and postharvest diseases of horticultural commodities. Crop Protection, Gueldford, v.25, n. 2. p.108-118, 2006.

CERQUEIRA, T. S.; JACOMINO A. P.; SASAKI F. F.; ALLEONI, A. C. C. Recobrimento de goiabas com filmes proteicos e de quitosana. Bragantia, Campinas, v.70, n.1, p.216-221, 2011.

CHIEN, P-J.; SHEU, F.;YANG, F-H. Effects of edible chitosan coating on quality and shelf life of sliced mango fruit. Journal of Food Engineering, Essex, v.78, n.1, p.225-229, 2007.

CHITARRA, M. I. F.; CHITARRA, A. B. Pós-colheita de frutas e hortaliças: fisiologia e manuseio. 2.ed. Lavras: UFLA, 2005. 785p.

DJIOUA, T.; CHARLES, F.; FREIRE JUNIOR, M.; FILGUEIRAS, H.; DUCAMP-COLLIN, M.-N.; SALLANON, H. Combined effects of postharvest heat treatment and chitosan coating on quality of fresh-cut mangoes (Mangifera indica L.) International Journal of Food Science and Technology, Oxford, v.45, p. 849-855, 2010.

DUTTA, P. K.; TRIPATHI, S.; MEHROTRA, G. K.; DUTTA, J. Perspectives for chitosan based antimicrobial films in food applications. Food Chemistry, London, v. 114, p. 1173-1182, 2007.

GONZÁLEZ-AGUILAR, G. A.; VALENZUELASOTO, E.; LIZARDI-MENDOZA, J.; GOYCOOLEA, F.; MARTÍNEZ-TÉLLEZ, M. A.; VILLEGAS-OCHOA, M.A.; MONROY-GARCÍA, I. N.; AYALA-ZAVALA, J. F. Effect of chitosan coating in preventing deterioration and preserving the quality of fresh-cut papaya 'Maradol.' Journal of the Science of Food and Agriculture, London, v. 89, n. 1 p. $15-23,2009$.
GTZ - DEUTSCHE GESELLSCHAFT FÜR TECHNISCHE ZUSAMMENARBEIT. Manual de exportación: frutas tropicales y hortalizas. Eschbom: GTZ, 1992. 34p.

HOJO, R. H.; JOSÉ, A. R. S.; HOJO, E. T. D.; ALVES, J. F. T.; REBOUÇAS, T. N. H.; DIAS, N. O. Qualidade de manga 'Tommy Atkins' pós-colheita com uso de cloreto de cálcio na pré-colheita. Revista Brasileira de Fruticultura, Jaboticabal, v.31, n.1, p.62-70, 2009.

JIANG, Y.; LI, Y. Effects of chitosan coating and postharvest life and quality of longan fruit. Food Chemistry, London, v. 73, p. 139-143, 2001.

LIU, J.; TIAN, S.; MENG, X.; XU, Y. Effects of chitosan on control of postharvest diseases and physiological response of tomato fruit. Postharvest Biology and Technology, Amsterdam, v.44, n. 3, p. 300-306, 2007.

MORAIS, P. L. D.; FILGUEIRAS, H. A. C.; PINHO, J. L. N.; ALVES, R. E. Ponto de colheita ideal de mangas Tommy Atkins destinadas ao mercado Europeu. Revista Brasileira de Fruticultura, Jaboticabal, v.24, n.3, p.671-675, 2002.

PLOTTO, A.; NARCISO, J. A.; RATTANAPANONE, N.; BALDWIN, E.A. Surface treatments and coatings to maintain fresh-cut mango quality in storage. Journal of the Science of Food and Agriculture, London, v.90, p.2333-2341, 2010.

SANTOS, C. A. A.; CASTRO, J. V. de; PICOLI, A. A.; ROLIM, G. S. Uso de quitosana e embalagem plástica na conservação pós-colheita de pêssegos 'Douradão'. Revista Brasileira de Fruticultura, Jaboticabal, v.30, n.1, p. 88-93, 2008.

SUN-HUA, C.; WEI-MIN, W.; XING-ZHOU, X. Study on effect of chitosan film coating on mango storage. Food Science and Technology, London, v.16, n.3, 2007.

ZHU, X.; WANG, Q.; CAO, J.; JIANG, W. Effects of chitosan coating on postharvest quality of mango (Mangifera indica L. Cv. Tainong) fruits. Journal of Food Processing and Preservation, Westport, v. 32 , n. 5 , p.770-784, 2008. 